\title{
Entrelacs
}

Cinéma et audiovisuel

15 | 2018

Récits de soi

\section{Le film mental}

\section{Benoît Labourdette}

\section{OpenEdition}

\section{Journals}

Édition électronique

URL : http://journals.openedition.org/entrelacs/3066

DOI : 10.4000/entrelacs.3066

ISBN : 2261-5482

ISSN : 2261-5482

Éditeur

Éditions Téraèdre

Référence électronique

Benoît Labourdette, «Le film mental », Entrelacs [En ligne], 15 | 2018, mis en ligne le 21 octobre 2018, consulté le 20 avril 2019. URL : http://journals.openedition.org/entrelacs/3066 ; DOI : 10.4000/ entrelacs.3066

Ce document a été généré automatiquement le 20 avril 2019.

Tous droits réservés 


\section{Le film mental}

\section{Benoît Labourdette}

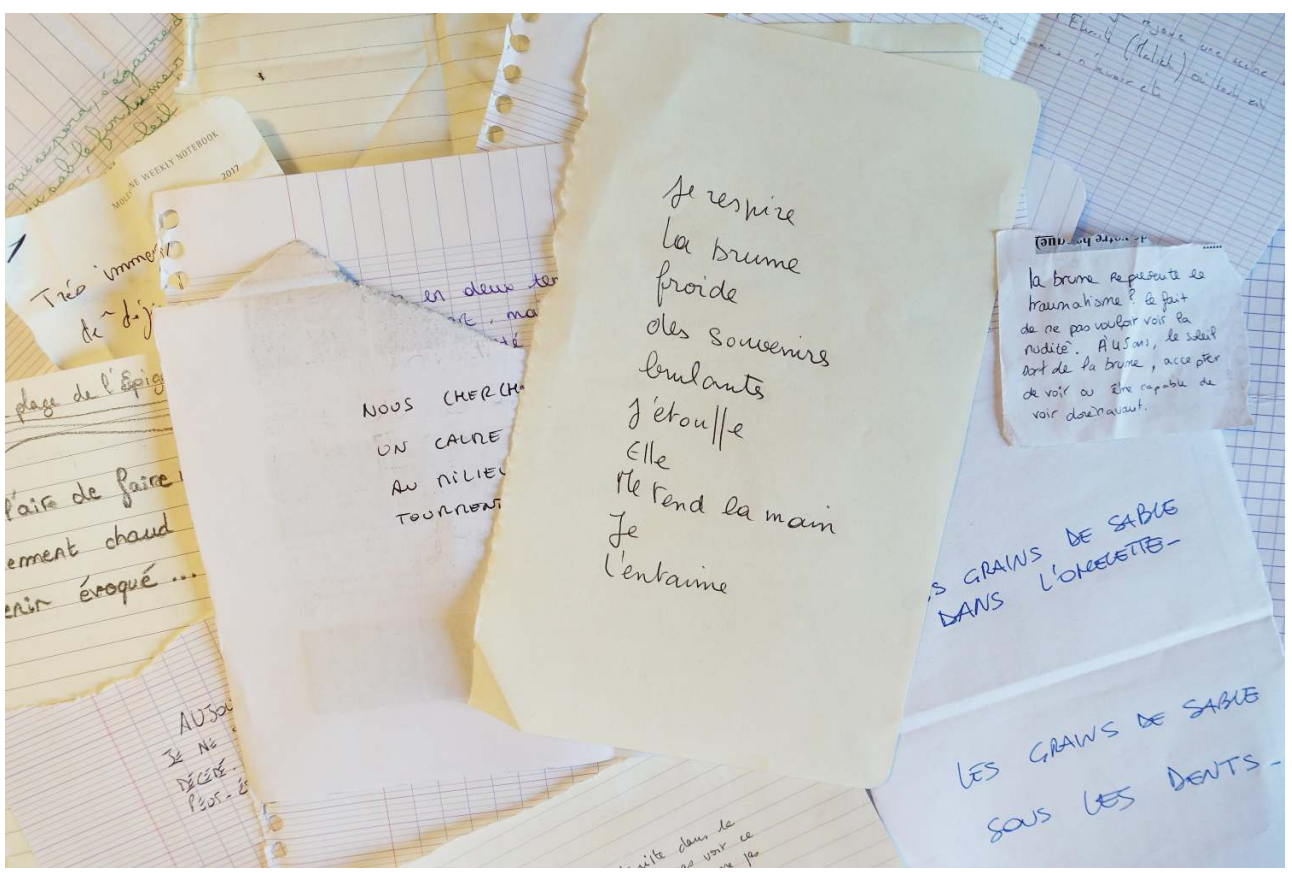

Photographie de Benoît Labourdette : Mots à l'origine du film collectif Plage de l'Espiguette (2017)

1 Si l'on se place du point de vue de la perception, qu'est-ce qu'un film ? Quelle est la différence entre un film vu et la réalité perçue ? Entre un rêve et un film ? Entre un film et un spectacle vivant ? Entre un film et un concert? Entre la radio et le cinéma ? Entre un roman et un film?

2 Ces questions peuvent étonner de prime abord et paraitre candides. Mais un film (par le truchement des techniques de l'image et du son) provoque avec son dispositif un ensemble de stimuli visuels et auditifs. Ceux-ci font réagir nos organes de perception (yeux et oreilles), qui produisent des signaux électriques, ensuite interprétés par notre cerveau comme étant des images animées et des sons, qui souvent nous racontent une histoire. 
Pour illustrer ceci par un exemple simple, nous savons bien que sur un écran de cinéma, d'ordinateur ou de télévision, il n'y a aucun mouvement. Il ne s'agit que d'une suite d'images fixes, qui s'enchaînent à une certaine fréquence $(24,25$ ou 30 images par seconde généralement). Le mouvement que nous percevons, qui nous semble tout à fait naturel, n'est en fait qu'une illusion que notre cerveau reconstitue à partir de cette suite d'images fixes. Ainsi le film, en tant qu'objet animé, n'existe pas à l'extérieur de nous, il est littéralement et physiologiquement créé par notre cerveau.

Par ailleurs des études récentes en neurosciences (j'ai lu cela dans Le Monde, je ne retrouve plus la référence) montrent que voir un film ou se faire raconter un film sont deux activités qui mobilisent à peu près les mêmes zones du cerveau. Ainsi, il n'est pas, du point de vue de la perception, de différence fondamentale entre regarder un film ou se le faire raconter. Tout comme lorsque nous ne savons pas faire la différence entre un souvenir vécu ou un souvenir reconstitué à partir de ce qui nous a été raconté.

Enfin, les stimuli reçus par nos yeux ne produisent, dans notre cerveau, pas forcément seulement des images et les stimuli reçus par nos oreilles pas seulement des sons. Alfred Hitchcock l'avait bien compris, quand, à l'époque de ses premiers films muets, il fabriquait des sons dans l'esprit du spectateur, en montrant une cloche qui tinte en gros plan, ou en filmant, à travers un plancher translucide, les pas du voisin du dessus, pour nous faire " entendre » des bruits de pas. Lorsqu'on en fait l'expérience, il est en effet troublant de ressentir à ce point des sons alors qu'on est pourtant devant une image muette.

6 En tant que cinéaste, il m'est apparu presque indispensable d'explorer le cinéma plus avant du côté de la perception, en inventant un nouveau dispositif, que j'ai nommé le film mental.

\section{Le vrai film est dans notre esprit}

7 Depuis une dizaine d'années, dans les nombreux workshops de réalisation de films que j'anime, je propose régulièrement aux participants, la veille pour le lendemain, de réaliser un film individuellement, tourné en plan-séquence, qui consiste à filmer ce qu'ils voient par une fenêtre de chez eux et à raconter, en voix-off et en direct sur cette image, un souvenir vécu important pour eux. Ces films sont visionnés collectivement le lendemain de leur tournage, c'est toujours une expérience de cinéma très forte. Les perceptions que je relate ici sont les miennes et celles de beaucoup d'autres spectateurs. J'ai commandé et vu plusieurs centaines de films de ce type. Ce qui m'a toujours surpris c'est à quel point les mots, l'histoire racontée en voix-off, produisaient chez les spectateurs bien plus d'images que l'image visuellement présente devant nos yeux. J'ai compris à travers ces films que la distinction « technique » entre image et son n'était pas du tout, dans la perception du spectateur, aussi clivée que cela. Dans ces films, les mots nous font voir plus d'images que l'image qui est devant nos yeux. J'en ai maintes fois fait l'expérience sensible. Des villes étrangères, des maisons familiales, des montagnes, des jeux d'enfants, des aéoroports et des vieilles villes... toutes ces images apparaissent dans le cadre de fenêtres vides.

8 On dit traditionnellement que le cinéma est l'art de montrer. Je dirais que le cinéma est l'art de faire naître des images et des sons dans l'esprit du spectateur. Les deux espaces (l'espace mental et l'espace physique) sont sans doute bien plus différents l'un de l'autre 
qu'on veut bien croire. Par ailleurs, on peut voir un film plusieurs fois, en y voyant à chaque fois des choses différentes. Cela vient confirmer le fait que le vrai film se situe dans l'esprit du spectateur bien plus que sur l'écran. Il n'est pas non plus dans la bobine, la cassette vidéo ou le disque dur, il est créé par notre esprit. Aussi, un film n'est pas le même pour chaque spectateur (il suffit de demander à plusieurs personnes de raconter le même film pour s'en rendre compte) et il peut varier pour la même personne en fonction des situations de réception. Une comédie par exemple n'a pas du tout le même impact dans une salle pleine que seul devant son écran, on ne rira pas aux mêmes moments.

Il y a aussi le cas de ce film des années 1990, Shazaam ${ }^{1}$ dont des centaines de personnes disent se souvenir, mais qui est introuvable dans les archives. A-t-il existé dans la réalité ? Assurément, mais on n'en trouve aujourd'hui pas trace ailleurs que dans la tête des spectateurs.

\section{L'intuition du film comme objet mental}

10 Reprenons la question initiale : qu'est-ce qu'un film ? C'est donc une perception, subjective et reconstruite par le cerveau, face à un écran qui nous propose des images et des sons. Mais quelle est la différence avec le spectacle vivant ? Dans le cas du spectacle vivant, nous ne sommes pas face à un écran mais face à des êtres humains, et nous ne ressentons pas le même type de choses. Ainsi, même si les perceptions peuvent parfois être proches, la différence essentielle du protocole de représentation artistique ne met pas notre appareil cognitif et sensible dans les mêmes dispositions. Il semble que nous ne mobilisions pas les mêmes régions de notre cortex cérébral face à un écran inanimé que face à au vivant. Heureusement !

11 Fort de ces réflexions nourries par mon expérience, lors d'une table ronde que j'ai animée en novembre 2016 sur la thématique de la voix-off, dans le cadre du Festival du film Autobiographique d'Olonne sur mer, j'ai eu brusquement l'intuition de tenter une expérience, afin de corroborer mes questionnements. Pendant que les intervenants parlaient, j'ai feuilleté le catalogue du festival, j'y ai trouvé un texte de présentation d'un film. J'ai alors inopinément proposé aux spectateurs, assez surpris, de faire ensemble un film, maintenant, tout de suite, tous ensemble, sans qu'il n'y ait rien sur l'écran. Ce film a été enregistré, il est ici : www.benoitlabourdette.com/ressources/conferences/festivaloff-table-ronde-sur-la-voix-off-et-realisation-de-film-collectif

12 J'ai expliqué aux spectateurs que j'allais faire la voix-off de ce film à venir, qu'il aurait un titre au début et un générique à la fin (dits par la voix-off). Eux devraient fermer les yeux. Je décrirai la première image du film, et ensuite ils devraient laisser cette image se transformer, au gré des images suggérées par la voix. Puis, après le générique de fin, tout le monde devrait rouvrir les yeux, et partager oralement avec les autres le film, singulier, qu'il aurait vu.

\section{Saint Malo, mon premier film mental}

Qu'il y ait un « vrai » film sur l'écran ou pas, de toutes façons chaque specateur crée son propre film. J'ai voulu expérimenter cela concrètement. Saint Malo est le titre du premier film mental, évoqué plus haut, que j'ai proposé dans une salle de cinéma. J'ai décrit aux spectateurs la première image du film. Cela allait être un plan fixe de la ville de Saint 
Malo. Pour ceux qui connaissaient, ils devaient se remémorer un endroit de cette ville et pour les autres l'imaginer. Puis j'ai demandé aux spectateurs de fermer les yeux, de voir en eux cette première image, pour la laisser se transformer au fil de l'écoute des mots. Dans cette salle de cinéma, devant un écran, ils étaient dans la posture de recevoir un film. Et j'ai lu ce texte, très lentement, en laissant du silence entre chaque phrase, entre chaque mot:

\section{Saint Malo}

On assiste au départ du trois mâts du port de Saint Malo, pour une campagne de six à sept mois au large du Canada. Les différentes tâches sont montrées : l'entretien du bateau, l'escalade des mâts pour la manoeuvre des voiles. La préparation des lignes et des appâts. Le retour des doris chargées de poisson. Le transfert du poisson dans la goëlette. Le dépeçage et le salage. Tempêtes. Icebergs. Froid glacial. Eprouvent les marins. La présence d'un navire hôpital leur permet de bénéficier de soins et de recevoir le courrier et le réconfort d'un aumônier.

Un film collectif, Olonne sur mer, 2016.

Enfin, j'ai proposé à ceux qui le souhaitaient de raconter au micro le film qu'ils venaient de voir, que leur esprit avait créé. Extraits des réactions orales :

«Je n'ai pas vu le film, et je peux vous dire que je me suis vraiment fait un film.

« Derrière mes yeux fermés, j'ai vu un montage alterné : je voyais Saint Malo, les bateaux, Saint Malo, les bateaux... en permanence. »

«Moi, ayant travaillé dans le milieu de la pêche, je suis resté dans le milieu de la pêche, plutôt une approche technique, j'ai vu ça comme un reportage. »

« Moi j'ai beaucoup navigué à Saint Malo, et le film m'a fait un espèce d'arrêt sur image. Je me suis arrêté au phare et je me suis demandé s'il existait à l'époque où partaient ces bateaux."

«Pour moi la voix-off était la voix d'une morue séchée arrimée à Saint Malo. »

« Des exercices comme ça, ça nous fait mieux comprendre. Là, c'était une voix-off qui évoquait des images, mais la voix-off ça peut être bien autre chose. »

«Ca m'a relié totalement au port.»

«Ca m'a fait prendre conscience de la force de son propre vécu, parce qu'en fait à Saint Malo j'ai une photo d'enfance sur la plage avec les fûts derrière, car à Saint Malo il y a une protection avec des bois noircis, et en fait j'essayais de m'évader de cette image pour aller sur les bateaux. "

26 « Au cinéma, une image évoque autre chose que ce que nous voyons à l'écran, et c'est ce que nous allons chercher. "

Les spectateurs décrivent réellement des images qu'ils ont vues dans leur esprit, et indiquent que les films avec images servent aussi à découvrir d'autres images que celles que l'on voit. C'est donc bien une expérience de cinéma qui a été vécue, car elle a été proposée comme telle. Si j'avais dit que je racontais juste une histoire, la puissance de l'évocation visuelle n'aurait peut-être pas été si forte. Finalement, le rituel de la salle, le grand écran dressé dans l'obscurité, je l'ai ici remobilisé par la parole. Par mes mots, j'ai invité les spectateurs à installer un grand écran dans leur esprit, puis à éteindre la lumière. Au fond, au niveau cognitif c'est la même chose de l'imaginer ou de le vivre. Ayant reçu ces retours de spectateurs, j'ai alors nommé cette expérience, le film mental, 
car l'expérience des spectateurs m'a confirmé que ce film existait réellement, même s'il n'avait pas d'existence en tant qu'objet matériel.

Aussi, nous percevons un film comme un film, même lorsque nous ne le regardons pas dans une salle de cinéma. C'est parce qu'il y a un protocole, titre au début, générique à la fin, qui nous met dans la posture de spectateur de film. C'est le « contrat de lecture », bien explicité par la sémiologie (Roger Odin).

J'ai ici reproduit le texte du film Saint Malo, ce qui fait que l'article que vous êtes en train de lire contient ce film, réellement. Ce n'est pas un scénario, c'est le film, qui peut donc être reproduit par une simple lecture, en étant à chaque fois différent, comme tout film. Cela n'est pas sans évoquer les tout premiers films de l'histoire du cinéma, dont il n'y avait qu'un seul original (on ne savait pas encore faire de copies), qui étaient donc tournés plusieurs fois pour qu'il puisse en exister plusieurs exemplaires. L'exemple le plus illustre étant The great train robbery (Edwin Stanton Porter et Wallace McCutcheon, Etats Unis, 1903), dont nous il nous reste plusieurs versions, mais il en a existé une multitude.

\section{Le cinéma, la psychanalyse et Dieu}

Le cinéma est né à peu près en même temps que la psychanalyse à la fin du XIXème Siècle. La posture du spectateur dans la salle noire est celle du rêve éveillé. L'un des grands moteurs de la cure analytique est le transfert, c'est à dire la projection sur le thérapeute d'un autre personnage, afin de revisiter et réinvestir autrement des événements passés. C'est à dire revivre le « film » de sa vie d'une nouvelle manière. Il y a des liens, profonds, entre la découverte de l'existence de l'inconscient, c'est à dire se qui se cache en nous pour mieux nous diriger et la découverte d'une technologie d'enregistrement mécanique des images et du son, le Cinématographe, qui semble redonner vie à ce qui a a disparu. Ces liens méritent à mon sens d'être travaillés et questionnés, pour en approfondir les potentialités et ainsi creuser l'outillage critique, qui est de plus en plus nécessaire je crois à mesure que les images deviennent de plus en plus omniprésentes.

31 Par ailleurs, l'un des grands appuis de la légitimation de l'existence de Dieu, dans le cadre de la construction des systèmes d'organisation religieux monothéistes de la culture Occidentale est le Saint Suaire de Turin. C'est un drap, enveloppé sur le Christ à sa mort, qui aurait réalisé sa photographie, produit la trace et généré son image par empreinte à l'instant de sa mort, preuve de sa résurrection, donc de l'existence de Dieu. Première photo de l'Histoire, origine du Temps (nous comptons le temps à partir de ce moment là), et première mystification par les images, bien-sûr. En effet, ce linge a été daté du Moyen Âge par la technique du carbone 14. L'homme face à une image est donc aussi, du fait de l'histoire de l'Occident, l'homme face à l'existence de Dieu. Ce qui a structuré notre monde actuel est, comme en psychanalyse, la projection qui fut faite sur cette image, bien plus que sa réalité (puisqu'elle n'est pas réelle, même si certains essaient encore d'en prouver la réalité, avec force théories notamment l'hypothèse d'un bombardement neutronique dû à un puissant séisme à Jérusalem survenu à l'instant de la mort du Christ...). 


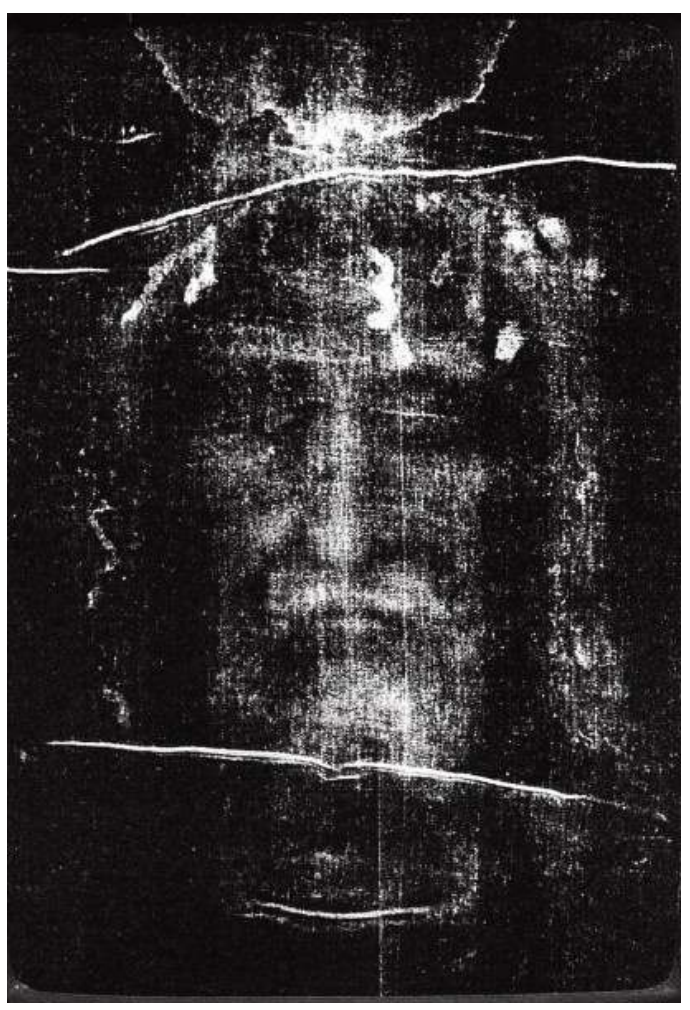

\section{Un Dieu numérique}

Le numérique forme une partie de plus en plus importante de la matière de nos vies. En effet, une grande part des échanges entre humains s'opère désormais par le truchement des technologies numériques, y compris cet article que vous êtes en train de lire. Le numérique est souvent vécu, et est vendu par le capitalisme comme un phénomène magique. L'expression cloud computing, littéralement « le calcul à l'intérieur des nuages » en est un bon exemple. Ainsi, l'image et son support principal actuel, le numérique, entraînent vers une pensée magique du monde, un simulacre, qu'il me semble essentiel de questionner. Une saine distance par rapport au monde et ses représentations se construit par des expériences de pensées singulières, décalées, ludiques, collectives, incarnées, vécues. La fonction de l'art, comme celle de la science, lorsqu'ils sont mis au service du progrès et non au service de la reproduction de l'ordre social, est de mettre en mouvement nos systèmes de représentations.

Ainsi, proposer à des spectateurs de regarder, concrètement, un film qui n'a pas l'existence technique d'un film mais qui pourtant en sera un, réellement, dans leur esprit et dans leur mémoire, est la méthode, ludique, d'une expérience singulière, d'un partage de subjectivités et d'un appui pour soutenir l'esprit critique dont nous avons bien besoin aujourd'hui, à l'ère d'un retour massif du religieux et du magique, via les technologies numériques. 


\section{Atelier pédagogique « Créer des images avec des sons} ॥

En parallèle à l'expérience du film mental Saint Malo, j'ai proposé avec l'ACRIF (Association des Cinémas de Recherche d'Île-de-France) un atelier en trois séances pour des lycéens nommé « Créer des images avec des sons », dont voici le texte introductif :

Proposer des exercices pratiques de créations sonores, en classe, sur des images cinématographiques, pour explorer le territoire de la perception, du fonctionnement $\mathrm{du}$ cerveau : comment les sons créent-ils des images mentales ? Comment les images que notre cerveau voit se construisent-elles en réalité par la conjonction de ce que nos yeux et nos oreilles nous transmettent selon leurs modes de traitement cognitifs ? Prendre conscience, par l'expérience, l'expérimentation, du fait que ce que nous voyons et entendons est fondamentalement l'objet de constructions mentales plus que de perceptions que l'on croit à tort objectives. Ainsi, aborder en profondeur, par les voies physiologique, créative et ludique, les pouvoirs de manipulation mentale que permettent les objets audiovisuels.

Dans ce cadre, le dernier exercice a consisté à faire créer, par des élèves face à leur classe, de courts films mentaux. Ce fut une séance de films très concentrée, très forte, dans le noir pendant deux heures. Le rituel du cinéma était pleinement opérant.

\section{Plage de l'Espiguette, mon deuxième film mental}

7 Dans le cadre de la journée d'étude intitulée «Les récits de soi. Outil et dispositifs du Je à l'écran ", organisée par Claire Chatelet et Julie Savelli (Université Montpellier 3) en avril 2017, dont cette publication est l'émanation, j'ai proposé, au sein d'une présentation que j'avais intitulée «Je ne le savais pas, mais je suis ma caméra », une nouvelle expérience de film mental. Le film est disponible ici :

www.benoitlabourdette.com/ressources/conferences/universite-montpellier-3-je-ne-lesavais-pas-mais-je-suis-ma-camera

Deux nuances par rapport aux premières expériences : d'une part le film est une improvisation, je raconte un souvenir d'enfance vécu dans un lieu proche de Montpellier (où se tenait la journée d'étude) et d'autre part le retour des spectateurs a été fait à l'écrit et non à l'oral. J'ai dit aux spectateurs que j'allais leur montrer mon dernier film, et que j'allais le créer en même temps. J'ai indiqué que la première image du film allait être une immense plage de sable fin, vide, avec de grandes dunes. La plage de l'Espiguette. J'ai aussi donné l'information qu'il paraît que le film Lawrence d'Arabie (David Lean, Etats Unis, 1962) aurait été tourné en partie sur cette plage. Une plage représentant le désert aride... Puis j'ai demandé aux personnes de fermer les yeux.

Voici le texte du film :

Plage de l'Espiguette*

Un film de Benoit Labourdette.

Aujourd'hui c'est la nuit. J'ai 45 ans. Je vais avec ma petite amie faire un pèlerinage plage de l'Espiguette. Il y a un pêcheur avec une grande canne à pêche. On discute avec lui. Et en vingt minutes la brume tombe. La brume est excessivement épaisse. Même à cinquante centimètres de 
distance on ne voit personne. Quelques lumières. Quelques lampes de poche. On est quatre, sur cette plage immense. Et je repense à ce que j'y ai vécu quand j'étais enfant.

\section{La brume}

Froide

des souvenirs

brûlants

J'étouffe

Elle

Me tend la main

$\mathrm{Je}$

L'entraîne.

51 Les grains de sable dans l'omelette. Les grains de sable sous les dents.

52 Nous cherchons tous

un calme immense

au milieu des

tourments du temps. 
61 reproduit, dans d'autres circonstances. Il suffit de le lire à haute voix à quelqu'un à qui on demande de fermer les yeux et d'imaginer un film dans sa tête.

62

Voici quelques photographies de ces mots, qui sont une trace de cette « version » du film Plage de l'Espiguette.
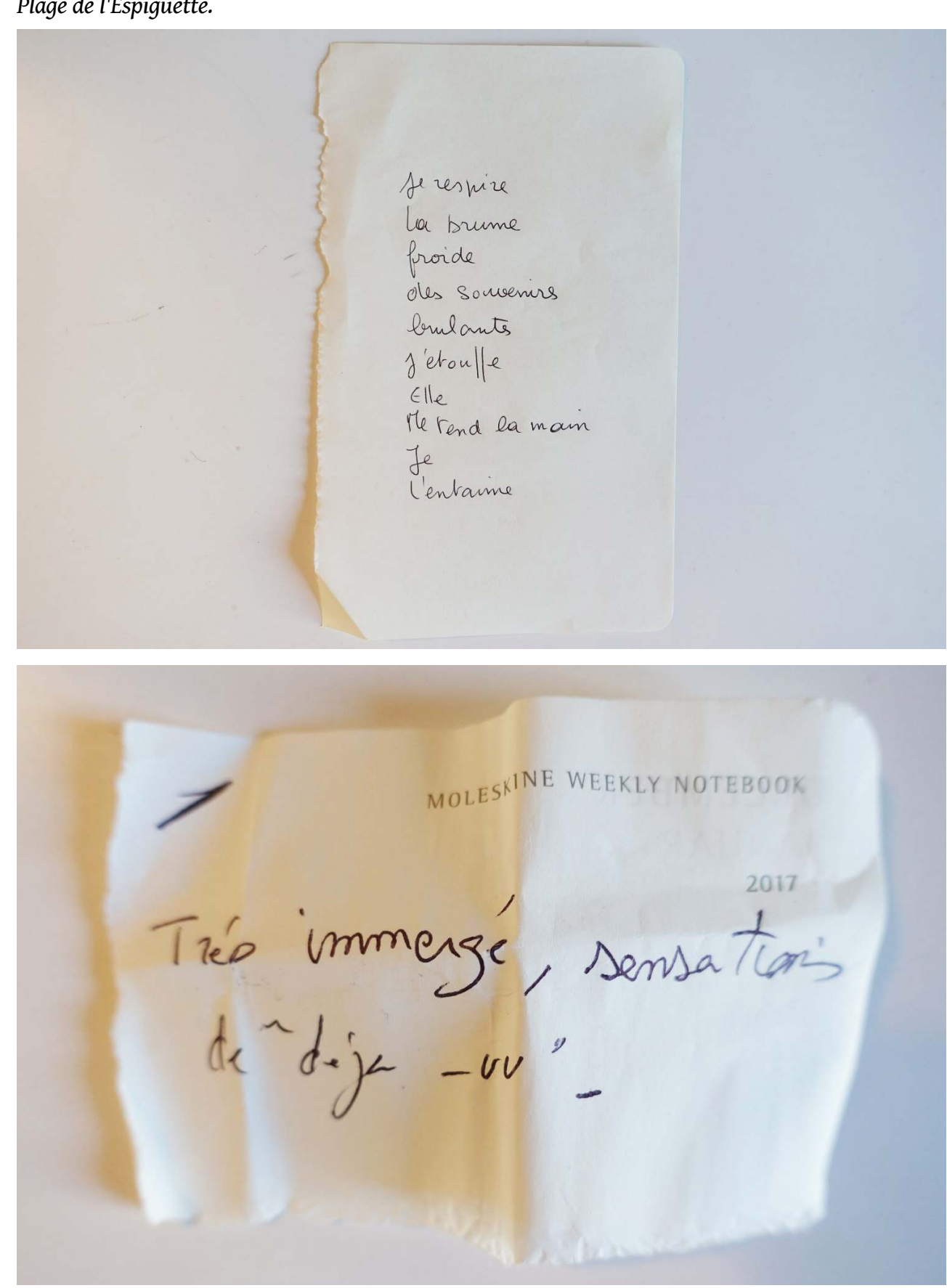

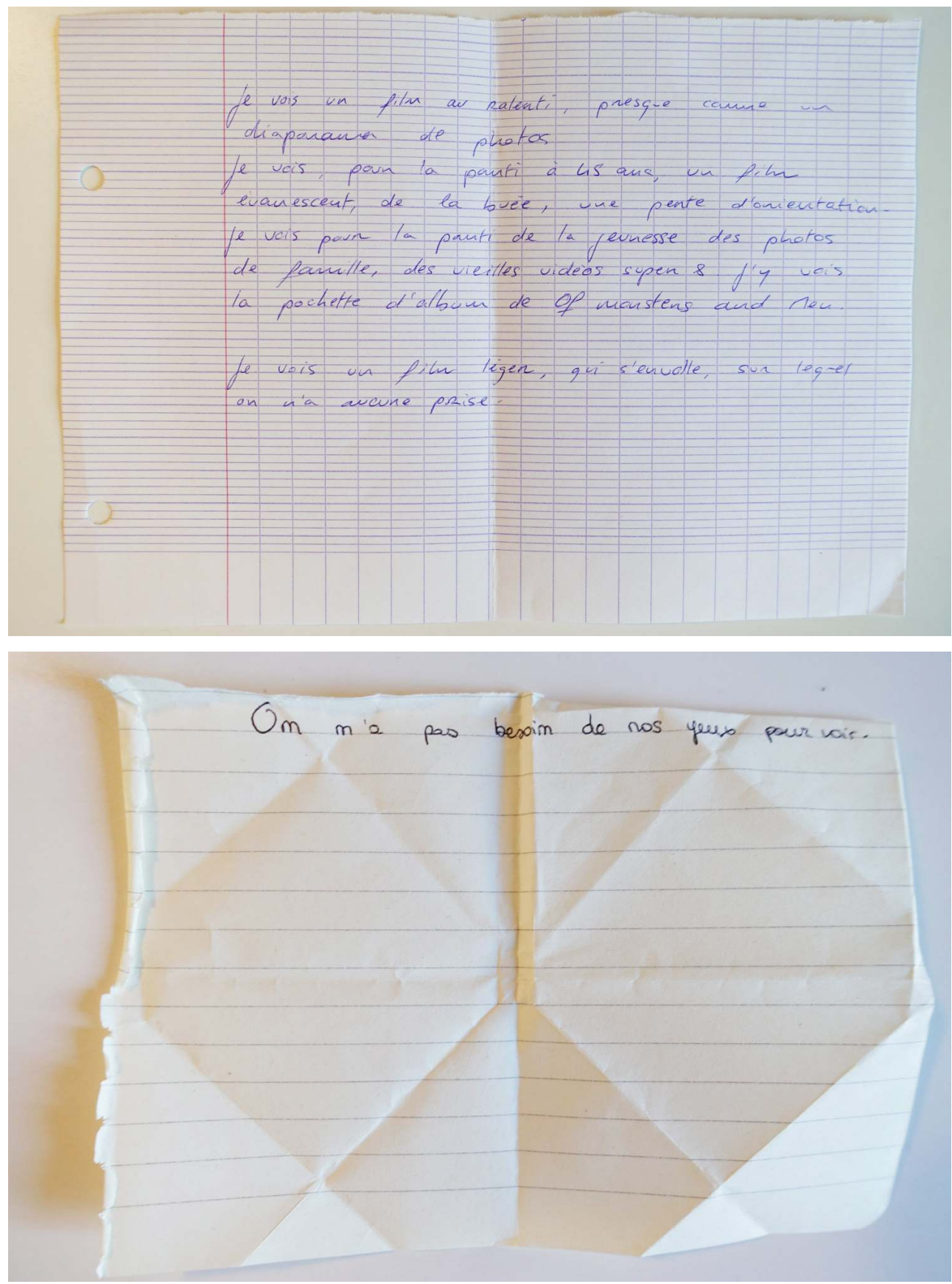
Avancer dans le brouillard, la nuit, avec une fimme que l'on aime, sur une plage que l'on a en souvenir déstabilisant, $n$ 'est-ce pas inserse-? Calme, lent, ca m'a repasé.

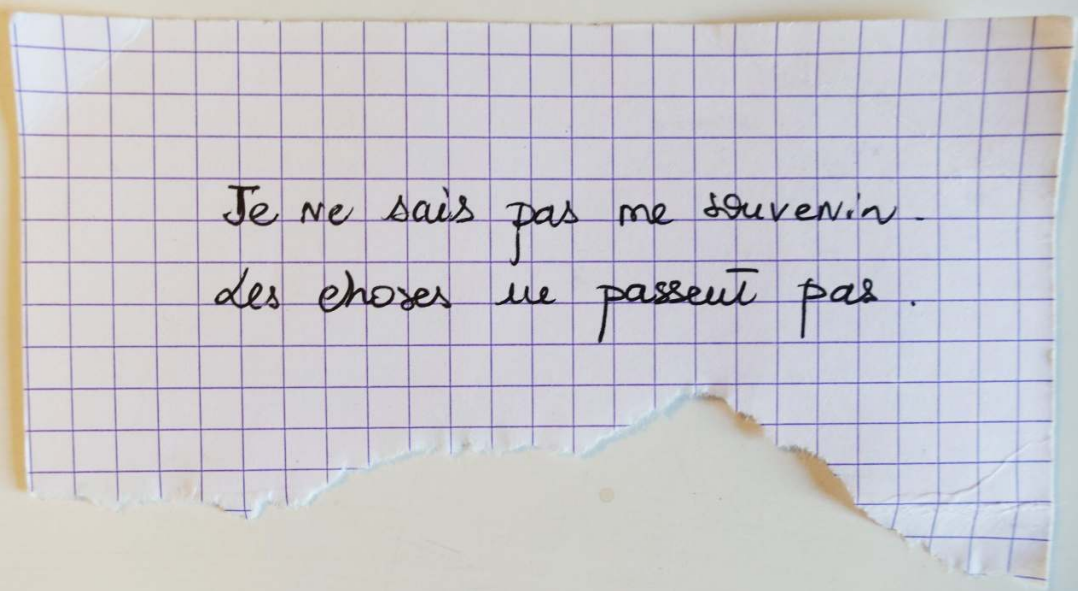

\section{NOTES}

1. 1. Sur Shazaam, voir : "Shazaam ", le film que tout le monde a vu... et qui n'a jamais existé Motherboard https://motherboard.vice.com/fr/article/ae7vm8/-shazaam--le-film-que-tout-lemonde-a-vu-et-qui-na-jamais-existe 
Ce film dont tout le monde se souvient... n'a jamais existé ! - Actus Ciné - AlloCiné http:// www.allocine.fr/article/fichearticle_gen_carticle $=18659263 . \mathrm{html}$

«Shazaam», le souvenir troublant d'un film inexistant - Le Temps https://www.letemps.ch/ societe/shazaam-souvenir-troublant-dun-film-inexistant

Tout le monde se souvient de ce film. Mais il n'a jamais existé https://www.nouvelobs.com/ rue89/rue89-sur-les-reseaux/20170105.RUE6100/tout-le-monde-se-souvient-de-ce-film-mais-iln-a-jamais-existe.html

Vous aussi, vous avez vu le film "Shazaam"? Impossible, il n'existe pas! - 7SUR7.be https:// www.7sur7.be/7s7/fr/31482/Le-meilleur-du-web/article/detail/3050201/2017/01/09/Vousaussi-vous-avez-vu-le-film-Shazaam-Impossible-il-n-existe-pas.dhtml

\section{RÉSUMÉS}

Du point de vue de la perception, un film n'est qu'une illusion formée par notre cerveau à partir de stimuli visuels et auditifs. Des images vues peuvent produire des sons et vice-versa. Chaque personne construit mentalement un film différent à partir du même film vu. Ainsi j'ai conçu une nouvelle proposition publique, le «film mental», un film qui n'existe que dans l'esprit du spectateur, un film qui n'a plus d'existence extérieure. Concepts philosophiques, physiologiques et retour d'expériences.

From the point of view of perception, a film is only an illusion formed by our brain from visual and hearing stimuli. Viewed images can produce sounds and vice versa. Each person mentally constructs a different film from the same film seen. So I designed a new public proposition, the "mental film", a film that exists only in the viewer's mind, a film that no longer has an external existence. Philosophical and physiological concepts then feedback of two experiences.

\section{INDEX}

Mots-clés : film mental, collectif, imaginaire, intuition, images mentales

\section{AUTEUR}

\section{BENOÎT LABOURDETTE}

Cinéaste, pédagogue, expert en nouveaux médias et en innovation culturelle. Il écrit et réalise fictions, documentaires, œuvres expérimentales et participatives. Sa démarche créative est pluridisciplinaire, il intervient aussi dans les champs de la peinture, du théâtre, de l'opéra, de la photographie, de l'architecture et de la musique. Il propose des innovations et anime des formations profesionnelles, dans le champ des nouveaux médias et de l'éducation à l'image. Il partage de nombreuses ressources sur : www.benoitlabourdette.com. 